\title{
Evolving use of real-world evidence in the regulatory process: a focus on immuno-oncology treatment and outcomes
}

\author{
Wagner ${ }^{1}$, Arvin Yang ${ }^{6} \&$ Mathias Hukkelhoven ${ }^{7}$ \\ ${ }^{1}$ Worldwide Health Economics \& Outcomes Research, Bristol Myers Squibb, Princeton, NJ, USA \\ ${ }^{2}$ Center for Observational Research \& Data Sciences, Bristol Myers Squibb, Princeton, NJ, USA \\ ${ }^{3}$ Informatics \& Predictive Sciences, Bristol Myers Squibb, Princeton, NJ, USA \\ ${ }^{4}$ US Health Economics \& Outcomes Research, Bristol Myers Squibb, Princeton, NJ, USA \\ ${ }^{5}$ Global Regulatory Strategy, Bristol Myers Squibb, Princeton, NJ, USA \\ ${ }^{6}$ Clinical Development, Bristol Myers Squibb, Princeton, NJ, USA \\ ${ }^{7}$ Global Regulatory \& Safety Sciences, Bristol Myers Squibb, Princeton, NJ, USA \\ *Author for correspondence: Tel.: +1 609302 3223; john.odonnell@bms.com
}

John C O’Donnell*,1, T Kim Le², Radu Dobrin ${ }^{3}$, Mitch Higashi ${ }^{4}$, Ashley Pereira ${ }^{5}$, Samuel

In recent years, regulatory bodies have increasingly recognized the utility of real-world evidence (RWE) for supplementing and supporting clinical trial data in new drug applications. Nevertheless, the integration of RWE into established regulatory processes is complex and the generation of 'regulatory-grade' real-world data faces operational, methodological, data-related and policy-related challenges. In parallel with this evolving role for RWE, immuno-oncology therapies have emerged as leading cancer treatments and are expected to continue to play a central role in the future. In this article, we review the current literature on the use of RWE for regulatory submissions, with a focus on novel anticancer immunotherapies, and discuss the utility and current limitations of RWE in the context of drug development and regulatory approvals.

First draft submitted: 8 June 2020; Accepted for publication: 29 September 2020; Published online: 19 October 2020

Keywords: drug development $\bullet$ immuno-oncology $\bullet$ real-world data $\bullet$ real-world evidence $\bullet$ regulatory approvals

Over the past several decades, increasingly complex drug approval processes overseen by authorities such as the US FDA and the EMA have necessitated concerted efforts to accelerate and reduce the cost burden of regulatory decision-making, which includes a move toward a defined integral role for real-world evidence (RWE) within regulatory approval processes [1-4]. Although data from randomized controlled trials (RCTs) remain standard for new drug evaluations, there are situations where a robust clinical trial is not practicable, for example, because of low recruitment prospects (e.g., for rare diseases), prohibitive anticipated costs and/or resource needs or ethical prohibition (e.g., in cases where there is no established standard of care [SoC] and an RCT would be unethical) [5-9]. Moreover, the clinical trial process is time consuming, which could delay availability of new effective treatments. In these circumstances, innovative approaches are needed to assess the benefits and risks of a medication. One such approach is to use real-world data (RWD) to help highlight unmet medical needs and demonstrate the effectiveness, tolerability and patterns of care of a given pharmacological agent in real-life settings and populations (Table 1) [2,3].

RWD sources are varied, and include electronic health records (EHRs), medical and pharmacy claims and billing, and product and disease registries, as well as patient-generated data (e.g., from in-home-use settings) [1,2]. Moreover, in the era of social media and availability and use of smartphones and tablets, data can be collected directly from patients using these tools. RWE represents information on usage patterns and potential benefits or risks of a medical product derived from analyses of these RWD [1]. By their nature, RWD can be more generalizable than those obtained from RCTs; in large part, this stems from the need for RCTs to control for variability [10] and to ensure strong internal validity, a requirement for robust efficacy assessment. By contrast, real-world studies may allow more permissive inclusion criteria, and accordingly may have fewer restrictions on patient characteristics and co-morbidity profiles, with commensurate enhancements in generalizability or external validity.

Future Medicine 
Table 1. Utility of real-world evidence in drug development and regulatory approvals.

\begin{tabular}{ll}
\hline $\begin{array}{l}\text { Stage of drug } \\
\text { development }\end{array}$ & Application of real-world evidence \\
\hline Preapproval & - Highlight diseases not currently served by existing treatments and current unmet needs \\
& - Help understand a potential role in the therapeutic approach \\
& - Facilitate clinical trial design and acceleration of novel therapies by characterizing patient populations in need \\
\hline $\begin{array}{l}\text { Peri-launch } \\
\text { (just before and }\end{array}$ & - Estimate treatment effect \\
immediately after & - Contribute to knowledge of patient safety \\
marketing approval) & - Inform clinical trial design and appropriate end point usage \\
& - Describe patient populations (e.g., characterize patients in underpowered subgroup analyses) \\
& - Act as external control for single-arm clinical trials
\end{tabular}

Questions about the effectiveness and safety of a new drug in real-world populations may be particularly important when clinical trial results lead to dramatic shifts in SoC for broad groups of patients. In the past 10 years, the advent of immuno-oncology (I-O) therapy has transformed the treatment landscape for numerous solid tumors and hematologic malignancies, and the emergence of novel agents harnessing the host immune system will likely continue on an upward trajectory for the foreseeable future [11-13]. In this constantly evolving environment, developers and regulators confront unique questions and drive critical developmental and regulatory processes that could be informed by RWE. In this article, we review current literature on the use of RWE in regulatory processes, with a focus on I-O therapy as a highly topical example of a relatively new and emerging class of treatments. We also discuss the utility and current limitations of RWE in the context of regulatory approvals and include select case studies to illustrate challenges of generating 'regulatory-grade' data from analyses of patients receiving I-O therapy in real-world settings.

\section{RWE \& regulatory assessments of new therapies}

Historically, the role of RWE in regulatory processes has been to supplement existing approvals, and not as a central component of initial drug applications. RWD were most frequently employed for postmarketing assessment of safety, for risk management or for assessment of benefit-risk across the drug life cycle [14]. RWE were also used to support new indications for approved medications; an early example from the I-O field being the 2013 EMA decision to extend the indication for ipilimumab (a cytotoxic T-lymphocyte-associated protein 4 inhibitor) to include previously untreated adults with unresectable or metastatic melanoma, which was based on data from both clinical trials and retrospective observational studies [15]. Infrequently, RWE has supported initial drug approvals, primarily in rare-disease and/or oncology settings. In these situations, RWD on historical clinical outcomes were drawn from chart reviews, expanded access programs, rare-disease registries and other clinical practice settings. Although not always the case, these RWD typically supported clinical data from interventional trials for which a control arm was infeasible or unethical and where a large effect size was expected based on initial data [1].

Early examples of RWE supporting new drug approvals include the 2006 and 2010 FDA accelerated approvals of Genzyme's alglucosidase alfa formulations for Pompe disease, a rare multisystem genetic disorder, which relied on comparison of clinical trial data with data from historical controls from a multinational disease registry [16,17]. In the oncology setting, an early example was the 2014 FDA accelerated approval of Amgen's blinatumomab for Philadelphia chromosome-negative relapsed or refractory B-cell precursor acute lymphoblastic leukemia, which included a comparison of data from a single-arm clinical trial with data from matched historical controls using propensity score methods [18-20]. More recent examples include the 2016 EMA approval of MolMed SpA's Zalmoxis, an immune-gene therapy for rare high-risk hematologic malignancies, which used historical data from the European Bone Marrow Transplantation patient registry [21], and the 2018 FDA approval of Fresenius Kabi AG's lipid emulsion fish oil triglycerides for pediatric patients with parenteral nutrition-associated cholestasis, which also used matched historical controls [22]. While this acceptance of RWE as supportive of new approvals is noteworthy, a frequent postmarketing requirement was that the clinical benefit be confirmed in an RCT [16,19,23].

Harnessing RWD to provide evidence for regulatory decision-making pose various operational challenges, including those related to feasibility (e.g., ease and cost of data access), governance (e.g., impact of the data source's data-sharing policy in relation to sponsored research) and sustainability (e.g., durability of data collection and analysis) [14]. Another operational challenge relates to governmental/regional policies that may limit data sharing and access to real-world patient data, such as the US Health Insurance Portability and Accountability Act and 


\begin{tabular}{|l} 
Table 2. Methodological and data-related challenges to the use of real-world evidence in regulatory decision-making. \\
Methodological \\
- Assignment of treatment based on physician judgment rather than randomized allocation may hinder establishment of a causal \\
relationship between treatment use and clinical outcome \\
- Unobserved confounders (e.g., physician choice, patient preference, unmeasured health risks or differential access to treatment) \\
- Absence of universally accepted methodological standards for study design and data reporting \\
- Lack of investigator expertise in real-world data generation and analysis \\
- Achieving sample size required to determine treatment efficacy \\
- Different behavior in real-world studies versus clinical trials (e.g., the Hawthorne effect) due to differences in monitoring frequency \\
- Logistics of engaging healthcare systems to collect usable real-world data \\
- Management of missing/incomplete data and follow-up \\
- Consistency and accuracy of data collection and recording (including data on potential confounders and effect modifiers) \\
- Possibility of data manipulation resulting from retrospective nature of data collection \\
- Risk of false positives as a result of under-reported multiple testing/reporting bias \\
- Establishing validity of patient-generated data (i.e., sources outside of the healthcare environment) \\
- Underestimation of patient safety concerns
\end{tabular}

the new General Data Protection Regulation in the European Union. Potential solutions to this challenge have been suggested, at least in the USA, including establishment of dedicated policy on patient privacy that would be overseen by the US government or an independent, nonprofit organization [24].

Even when operational barriers are overcome, generation of RWE is subject to various methodological and data-related challenges, as summarized in Table 2. A major limitation of RWD is that assignment of treatment based on physician judgment rather than randomized allocation may hinder establishment of a causal relationship between treatment use and clinical outcome [1]. Unobserved confounders (e.g., physician choice, patient preference, unmeasured health risks or differential access to treatment) can also influence outcomes of real-world research, whereas these factors are generally addressed by randomization in traditional clinical trials [3]. Additionally, under certain circumstances, it can be challenging to achieve a suitable real-world sample size to allow robust statistical analysis [25]; this is notable in oncology, where treatments increasingly target smaller subsets of patients, often defined by a genetic mutation. Other limitations of RWE include but are not limited to the challenges of identifying and defining reliable clinical end points in claims data, lack of follow-up and reliability in patient registries, and missing/incomplete data [26]. Indeed, the need to account for missing/incomplete data is a common challenge in real-world studies, both in the context of data that should be available but are often missing (e.g., performance status) and data that are generally not collected in routine clinical practice (e.g., history of co-morbidities, date of disease progression, etc.). The resultant level of uncertainty in terms of patient history is a particular issue for EHRs and administrative records, where there may be less granularity on the phenotypic status of patients, thus necessitating assumptions or proxy measurements to infer disease status [3].

There is increasing recognition of the need to address these limitations in order to increase confidence in RWE and ensure that the evidence is of 'regulatory-grade' quality. This includes research to develop specific real-world end points, such as a composite real-world mortality end point, a potential proxy for overall survival (OS) [27] and efforts to establish a systematic approach to improve comparisons between randomized trials and observational studies [28,29]. Additionally, this may involve larger initiatives designed to develop rigorous study designs and statistical methodologies and to standardize methods, several of which are underway in Europe and the USA. For example, the FDA-funded DUPLICATE initiative aims to replicate the results of RCTs using RWD, such as from claims databases, with the ultimate objective of exploring whether RWE aligns with clinical trial data [30,31]. Another, the Innovative Medicines Initiative, a public-private consortium comprising industry, academia, Health Technology Assessment agencies and regulators, has launched the GetReal (2013) and GetReal Initiative (2018) projects with the overall aim of assessing how robust new methods of RWE collection and synthesis could be developed and considered for adoption earlier in pharmaceutical research and development and the healthcare decision-making process [32]. Finally, the establishment of a pan-European integrated and sustainable network of real-world databases - the Data Analysis and Real World Interrogation Network (DARWIN) - that will access and analyze healthcare data of verified quality and content has been identified as a top priority by the joint Big Data Task Force of the EMA and the Heads of Medicines Agencies network [33]. With increased understanding of the potential value of RWE in driving regulatory decisions, these efforts and initiatives offer opportunities to highlight related challenges and potential solutions. 


\section{Impact of RWE on regulatory policy}

In 2016, the 21st Century Cures Act stipulated that the FDA considers the use of RWE in support of new indications for approved drugs and biologics, or to help address postapproval requirements. This was followed by the 2018 publication of the Framework for FDA's Real-World Evidence Program [1]. This program seeks to assess the utility of RWD for supporting changes to approved indications or assessing comparative effectiveness and safety of a licensed drug. The program will focus on addressing three key issues: whether the RWD are fit for the intended application; whether the trial or study design used to generate the RWD provides adequate scientific evidence to answer or help answer regulatory questions, and whether the study conduct meets FDA regulatory requirements, such as for study monitoring or data collection [10]. As a follow-up, the FDA published additional guidance for industry in 2019 on the process for submitting RWE as part of regulatory filings [34]. The 2018 FDA framework and subsequent guidance for industry represent nonbinding recommendations put forward while the FDA continues to explore approaches to optimize the utility of RWE. In Europe, the EMA and European Commission initiated the Adaptive Pathways approach in 2014 as a mechanism for allowing RWD to support regulatory submissions, and related guidance for industry followed in 2016 [35,36]. European regulators also continue to work toward integration of RWE within the existing regulatory system through ventures such as the aforementioned Innovative Medicines Initiative and DARWIN platform [37]. Importantly, external stakeholders from academia and industry will be instrumental to the FDA and EMA in determining whether RWE can be applied as a tool for future primary decision-making $[2,10]$. Further draft guidance on using RWE in the FDA regulatory process is expected to be published in December 2021 [38].

Although regulatory bodies are beginning to establish frameworks and guidance for use of RWE in their decisionmaking, and efforts are underway to formalize standards and methodology for generating RWE, there remains a lack of clarity on what constitutes 'regulatory-grade' RWE. The importance (and difficulty) of generating acceptable evidence has been highlighted in recent negative feedback from the FDA on some RWE submissions, centering on criticisms of the representativeness of real-world populations, data quality and potential bias (re: small sample sizes, missing/incomplete data, confounding factors, misclassification issues and selection bias) [38]. This may be further compounded by possible differences in definitions of 'regulatory-grade' depending on the end user (e.g., data for analysis of real-world clinical effectiveness vs data for real-world economic analysis) and the hypothesis, based on early results from the DUPLICATE initiative, that utility of RWE for replicating clinical trial results may depend on the therapy area and comparators utilized [31]. This lack of clarity on 'regulatory-grade' RWE stems, at least in part, from regulatory approvals and rejections that are nearly always communicated with no direct indication of the relative importance of the provided evidence in that decision. A recent example of this can be seen with the successful extension of the approved indications for palbociclib to include male patients with advanced or metastatic breast cancer. Although the associated multidiscipline review document and a related publication provided useful feedback on the strengths and weaknesses of the performed real-world analyses, highlighting several methodological limitations and related concerns regarding definitive evidence [39,40], the documents ultimately conclude that the RWE were supportive of the submission. Thus, while the submission was successful, it is unclear whether the submitted RWE was considered 'regulatory-grade' or whether it was necessary at all (i.e., the submission would have been approved without it). Indeed, experience suggests that the current RWE submission process may be more a case of 'policy by precedence versus policy by direction' - the industry draws on the apparent merits of previous submissions, rather than being specifically directed by the regulatory bodies to provide the information they require. This suggests a need for increased communication between regulatory bodies and those submitting data packages, particularly regarding exactly what is required from the RWD for the related evidence to inform their decisions. Moreover, these discussions will also provide a forum for agreement on other aspects related to quality of submitted RWE, for example, what criteria need to be met to establish a synthetic control or comparator arm using RWD. There is hope that future guidance from the regulatory bodies, building on the existing frameworks, will provide much-needed clarity on what is required for RWE to be considered 'regulatory grade.'

\section{RWE \& the regulatory assessment of I-O agents}

Immunotherapy for cancer is a rapidly expanding therapeutic area, as demonstrated by the $91 \%$ increase in the number of active or investigational I-O agents introduced worldwide and 31 FDA drug approvals over the past 2 years [13]. Immune checkpoint inhibitors targeting the PD-1/PD-L1 axis, for example, have become SoC for no less than 16 cancer types and tissue-agnostic indications [41]. Compared with conventional treatments, immune checkpoint inhibitors have demonstrated significantly greater efficacy and durability of response in patients with 
a wide range of cancers [42]. It is also evident from reported development pipelines that the number of I-O agents will further increase; for example, more than $1800 \mathrm{I}-\mathrm{O}$ agents are currently in development in the USA alone [13].

The fast-changing and expanding I-O treatment landscape highlights the need to better inform treatment decisions through rapid assessment of how new therapies affect patient outcomes. As discussed previously, clinical trials are the established mechanism for such assessments, but they can take a long time to conceive and conduct. Indeed, while the usual length for a Phase III trial is 1-4 years [43], many clinical trials experience significant delays to recruitment, which often leads to a further increase in the length of the study [44]. Since many new I-O agents address significant unmet medical needs, the time required to develop a trial protocol, recruit patients and conduct a clinical trial might be seen as an unacceptable delay to patients accessing new and effective treatments. Moreover, clinical trials for orphan drugs (i.e., those targeting rare diseases), where unmet medical needs may be greatest, on average take nearly twice as long as trials of nonorphan drugs [45]. This is a major consideration as I-O therapies are increasingly being extended to smaller patient populations with rarer conditions. Even in the case of less-rare cancers, recruitment into later-stage I-O studies (i.e., Phase III RCTs) can be challenging as data from earlier studies may be robust enough that investigators feel that clinical equipoise has been lost and are reluctant to enroll into larger trials where SoC has already been surpassed. In addition, patterns of response with I-O therapies are somewhat distinct from other cancer treatments and are often characterized by durable tumor suppression and associated long-term survival. This has led to calls for innovative methodologies and study designs [46,47], suggesting that traditional clinical trial approaches may not be optimal for assessing long-term effects of I-O therapy. A further consideration is the large number of emerging I-O agents [13]. This rapid expansion may result in a need to compare new agents and new combination regimens against each other and/or against an expanding list of SoC treatments. However, conducting multiple RCTs to facilitate these comparisons may be cost and resource prohibitive. In this environment, there is a strong case for increased acceptance on the part of regulatory bodies to submissions based on limited clinical trial data supplemented with RWD.

Over the past few years, there have been multiple examples of RWD sources rapidly providing valuable data on the impact of newly licensed I-O therapies, particularly in the context of assessing the generalizability of effectiveness and/or tolerability to broad patient populations [48-53], or specific populations usually excluded from clinical trials $[50,54,55]$. These postapproval data extend our overall understanding of the efficacy and safety profiles of new I-O therapies. However, as discussed earlier, the increased general acceptability of RWE in the context of regulatory filings and the expected need for RWD to contribute to I-O regulatory submissions indicate an important preapproval role for RWE in the emerging I-O field. In the next section, we present four illustrative cases in which RWE on I-O treatments and outcomes were used to support regulatory submissions. By necessity, we were compelled to focus on those cases with publicly available information on both the analyses and the associated regulatory submission and, as such, we may have omitted other relevant examples. Nevertheless, the following cases, which we consider to be appropriate 'archetypal' use cases highlight some of the common methodological and data-related challenges associated with the use of RWE in the regulatory process.

\section{Using RWE for an initial drug approval}

In September 2017, the European Commission granted marketing authorization for the PD-L1 immune checkpoint inhibitor avelumab as monotherapy for adults with metastatic Merkel cell carcinoma (MCC), primarily on the basis of data from the single-arm JAVELIN Merkel 200 clinical trial [56-58]. Before approval of avelumab, there was no established SoC for patients with this rare aggressive skin cancer, and most received one of several available chemotherapy regimens. Although JAVELIN Merkel 200 indicated the clinical activity of avelumab in metastatic MCC, the trial did not include a comparator arm. As such, a retrospective, observational study was conducted to provide data on outcomes of patients with metastatic MCC receiving chemotherapy regimens in clinical practice, which could then be compared with JAVELIN Merkel 200 data [56].

The observational study included 34 patients in Europe and 87 in the USA, with the latter divided into two cohorts comprising 67 patients who received first-line chemotherapy and 20 who received second- or later-line chemotherapy [56]. Naive, unadjusted clinical outcomes data from these patients were then compared with outcomes data from the JAVELIN Merkel 200 trial [59].

There were several methodological challenges associated with the comparison of data from JAVELIN Merkel 200 and the observational study. First, sample sizes were small, with 88 patients in the clinical trial and 67 first-line and 54 second- or later-line patients in the observational study. Second, while the patient populations were generally comparable, there were noteworthy imbalances. For example, 56\% of patients in JAVELIN Merkel 200 had an 
Eastern Cooperative Oncology Group performance status (ECOG-PS) of 0 and $44 \%$ had an ECOG-PS of 1. Among patients with available ECOG data in the observational study, only $21 \%$ had an ECOG-PS of 0 and $12.5 \%$ had an ECOG-PS of 2 or 3. Furthermore, the observational study included immunocompromised patients (22\% were immunocompromised) who were excluded from JAVELIN Merkel 200 [56]. It is noteworthy that the observational study population was split into 'immunocompetent' and 'all-qualified' subgroups for comparison with JAVELIN Merkel 200 data, which helped assuage concerns about the impact of immunosuppression on efficacy outcomes. However, this process further reduced the size of comparable samples, likely further impacting statistical reliability. An additional limitation relates to missing/incomplete data in the observational study. For example, 15 of the 87 US patients $(\sim 17 \%)$ and all 34 of the European patients had no ECOG-PS data. Interestingly, regression analyses requested in relation to a subsequent technology appraisal by the UK's NIH and Care Excellence appeared to suggest that no patient characteristics were prognostic of outcomes among these patients with metastatic MCC. As such, the sponsor concluded that there was no need to adjust for prognostic factors or to use alternative methods, such as conducting a matching-adjusted indirect comparison [60]. However, the NIH and Care Excellence review team raised concerns around drawing conclusions based on statistically nonsignificant results from these regression analyses as they could arise due to small patient numbers rather than the absence of a prognostic effect [60].

Despite the challenges noted above, the use of historical data for unadjusted comparisons with clinical trial data was accepted as the only solution to the lack of a comparator arm in JAVELIN Merkel 200 and the difficulty in conducting a dedicated RCT in such a rare condition. Ultimately, these real-world analyses were recognized by the EMA as supportive of the avelumab submission [56].

\section{Using RWE to support a new indication}

The PD-1 immune checkpoint inhibitor nivolumab was initially approved by the FDA in 2014 for second- or laterline treatment of unresectable or metastatic melanoma. Over the following years, the FDA approved nivolumab for several additional indications, including renal cell carcinoma and non-small-cell lung cancer. In August 2018, the FDA granted accelerated approval for nivolumab use in patients with small-cell lung cancer (SCLC) who had progressed after platinum-based chemotherapy and $\geq 1$ other line of therapy, primarily on the basis of clinical outcomes from the SCLC cohort of the CheckMate 032 clinical trial [61]. Before this approval, patients with SCLC failing $\geq 2$ therapies typically received SoC chemotherapy. As CheckMate 032 did not include a SoC comparator arm, a population-adjusted indirect comparison analysis was performed to provide data on the effectiveness of nivolumab (with or without ipilimumab) versus SoC treatment approaches (i.e., non-I-O regimens) for patients treated with two prior lines of chemotherapy or chemoradiotherapy (i.e., third-line) for SCLC [62,63].

For this analysis, two comparator cohorts were constructed from a US-based real-world database that matched the inclusion/exclusion criteria of CheckMate 032. One cohort included patients receiving a third-line therapy who had not received an I-O agent in any line $(\mathrm{n}=78)$, while the other cohort included patients receiving a third-line therapy that was not an I-O agent, but who had received I-O therapy in a previous line $(\mathrm{n}=92)$. From CheckMate 032, individual patient data were extracted only from patients with two prior lines of therapy and who received third-line therapy with nivolumab alone $(n=78)$ or nivolumab plus ipilimumab $(n=43)$ [63]. In the main analysis, a regression-based indirect comparison was performed using a Cox regression model for nivolumab (with or without ipilimumab) and modeling OS using individual patient data from CheckMate 032. This model was used to predict survival outcomes for nivolumab (with or without ipilimumab) that would have been observed in the real-world database for comparison with outcomes for patients receiving SoC treatments. In addition, a matching-adjusted indirect comparison was performed as a sensitivity analysis, using a propensity score function.

Several methodological and data-related challenges were associated with this analysis. For example, sample sizes for each cohort were relatively small, resulting in substantial uncertainty in relation to the OS curves where numbers at risk were very small at later time points. In addition, the analysis methodology involved adjusting models for potential prognostic factors, which were identified via a targeted literature review; however, of the factors identified, only age, sex, race, smoking status, disease stage, platinum sensitivity and ECOG-PS were reported in both the CheckMate 032 and real-world cohorts, and thus could be considered for inclusion. Moreover, to prevent overfitting the multivariate models given the small sample sizes, the final model was adjusted for only four factors: sex, disease stage, platinum sensitivity and ECOG-PS. As such, there was a level of uncertainty regarding the potential influence of any unknown or unmeasured prognostic factors on survival; for example, information was not available on the presence of liver or brain metastases, or on prior surgery or radiotherapy. Even those factors included in the final model were reported in the real-world cohort with varying frequency; for example, while all 
patients had information on sex and more than $95 \%$ had information on platinum sensitivity, a third of patients in the real-world database had no reported ECOG-PS.

Despite these challenges, the methods used for this analysis were considered the best attempt to account for between-study differences given the difficulty in evaluating comparative efficacy on the basis of a single-arm trial and in the absence of an RCT. Moreover, data from this analysis supported the clinical trial data from CheckMate 032 used to secure the new indication approval of nivolumab as a third-line treatment for metastatic SCLC. Of note, in a more recent Phase III clinical trial, nivolumab monotherapy did not provide a survival benefit over chemotherapy (topotecan or amrubicin) for patients with SCLC who progressed after first-line platinum-based chemotherapy, although the safety profile strongly favored nivolumab [64]. However, current guidelines for secondor later-line treatment of SCLC in the USA still include nivolumab \pm ipilimumab (based on the CheckMate 032 data) and have also recently been expanded to include pembrolizumab [65], suggesting continued confidence in using PD-1 immune checkpoint inhibitors in later lines of SCLC therapy.

\section{Using RWE in support of a proposed indication expansion across geographies}

By late 2017, data from the randomized, double-blind, Phase III ATTRACTION-2 clinical trial had demonstrated significant clinical benefit of nivolumab over placebo for heavily pretreated Asian patients with advanced or metastatic gastric or gastroesophageal junction cancer (GC/GEJC) [66], leading to regulatory approval in Japan [67]. At the same time, data from the Phase I/II CheckMate 032 trial had indicated clinical activity of nivolumab monotherapy as third- or later-line therapy in patients with GC/GEJC or esophageal cancer from Europe and the USA [68]. While the CheckMate 032 results suggested similar efficacy for nivolumab in Western patients with GC/GEJC, there remained a need to bridge the placebo-controlled clinical trial data in Asian patients to a Western population. On this basis, a retrospective, observational study was conducted to evaluate baseline characteristics, survival outcomes and duration of therapy in US real-world SoC cohorts matched to patients with GC/GEJC who received $\geq 2$ prior lines of therapy in the ATTRACTION-2 placebo arm or the CheckMate 032 nivolumab monotherapy arm [69].

In order to match patients from a US real-world database to the ATTRACTION-2 and CheckMate 032 arms, patients were identified who met inclusion/exclusion criteria similar to those used for ATTRACTION-2 and CheckMate 032. This was followed by frequency matching to align baseline characteristics in the real-world cohorts with the trial arms, with a focus on characteristics identified as significantly associated with OS in univariate analyses [69]. Figure 1 shows a schematic of the inclusion/exclusion criteria alignment and frequency-matching processes that resulted in the matched real-world cohorts used for the subsequent comparisons [69].

In addition to relatively small sample sizes, the main challenge for this comparison of RWD with data from ATTRACTION-2 and CheckMate 032 was related to the population-matching process. First, the initial step of this process had to be based on a relatively limited set of trial-specific inclusion/exclusion criteria. Moreover, while the subsequent frequency-matching process was successful in aligning the populations with respect to most patient characteristics, there were some noteworthy differences. For example, compared with patients in the ATTRACTION-2 placebo arm, patients in the matched real-world cohort had a higher median age ( 66 vs 61 years), a higher proportion had a primary tumor in the gastroesophageal junction (23 vs $7 \%)$ and a lower proportion had received $\geq 3$ prior therapies (44 vs 82\%) [69]. Similarly, compared with patients in the CheckMate 032 nivolumab arm, patients in the matched real-world cohort had a higher median age (64 vs 58.5 years) and a lower proportion had received $\geq 3$ prior therapies ( 40 vs $57 \%$ ). Univariate analyses assessing the influence of factors on OS indicated that only ECOG-PS (0 vs 1), disease stage (stage IV vs other stages) and site of primary tumor (GC vs GEJC) significantly impacted OS [69]. This suggested that the only imbalance likely to influence the comparisons would be the lower proportion with a primary tumor in the gastroesophageal junction in the real-world cohort matched to the ATTRACTION-2 placebo cohort. However, based on the relatively small real-world samples in this study, it is possible that differences in age and the number of prior therapies did influence survival outcomes, despite the lack of a statistically significant association with OS in the univariate analyses. Moreover, patient medical histories for the real-world cohorts were often incomplete and may have been inaccurate (e.g., due to misclassification and incomplete data entry), further challenging the veracity of this matching process.

While considering the limitations of the frequency-matching process, the comparisons performed were considered at the time the only viable method for bridging the Asian clinical trial data to a Western population in the absence of an RCT in Western patients. Although available RWE was included in the regulatory-marketing applications, it 


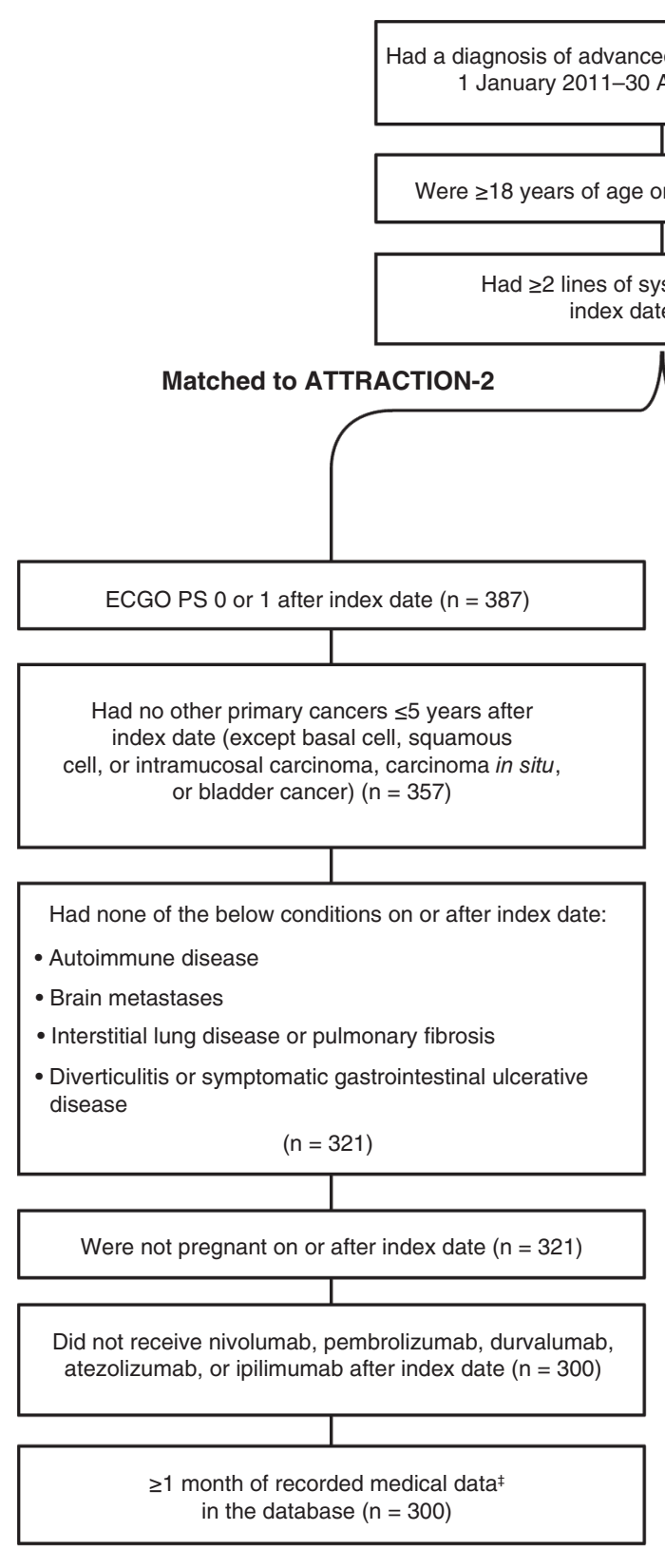

RW ATTRACTION-2 main cohort: $\mathbf{n}=\mathbf{3 0 0}$

- Frequency-matching criteria: distributions of initial diagnosis of disease stage (stage IV vs other), ECOG PS (0 vs 1), and primary tumor location (GC vs GEJC)

\section{RW ATTRACTION-2 matched cohort: $\mathbf{n}=\mathbf{9 0}$}

Matched to CheckMate 032

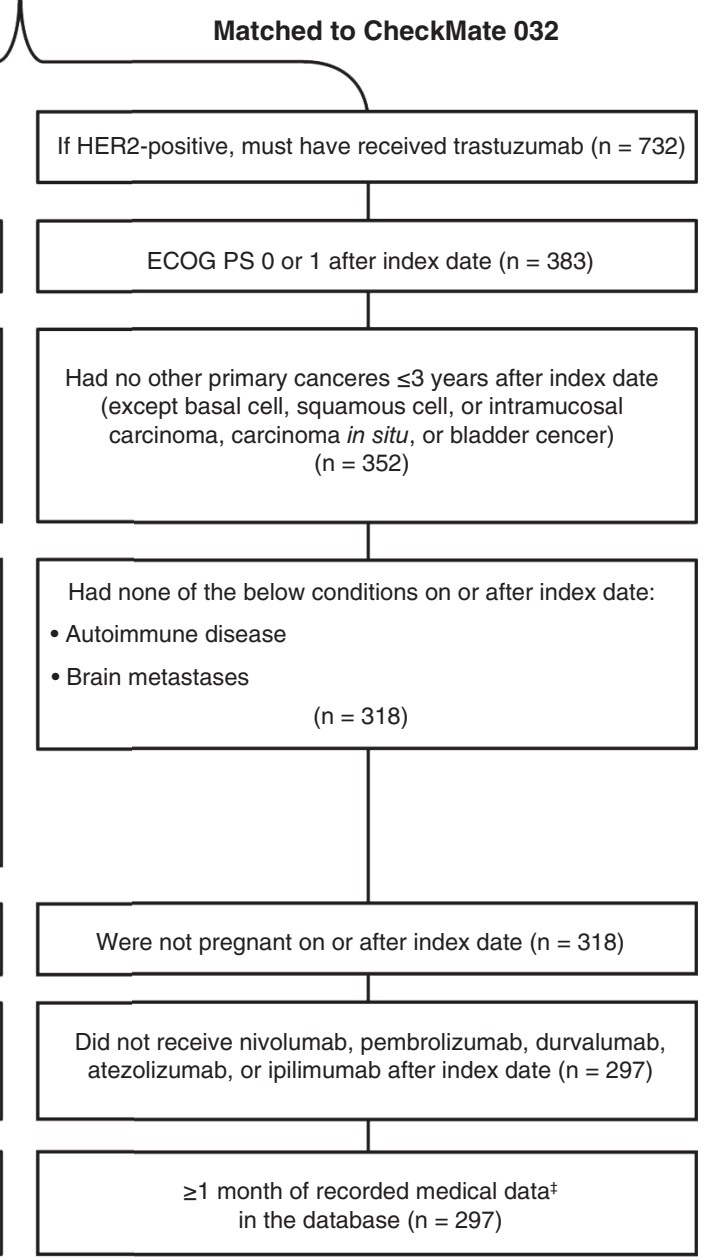

\section{RW CheckMate 032 main cohort: $\mathrm{n}=297$}

- Frequency-matching criteria: distribution of ECOG PS (0 vs 1) and primary tumor location (GC vs GEJC)

RW CheckMate 032 matched cohort: $n=100$

Figure 1. Inclusion/exclusion criteria alignment and frequency matching for patients in US real-world standard of care cohorts matched to the ATTRACTION-2 placebo and CheckMate 032 nivolumab monotherapy arms.

$\dagger$ Excluded patients who were enrolled in clinical trials.

¥Defined as data from outpatient physician office visits, nonfacility visits, laboratory visits, treatment/procedure visits or medication administration.

ECOG PS: Eastern Cooperative Oncology Group performance status; GC: Gastric cancer; GEJC: Gastroesophageal junction cancer; RW: Real world.

This figure was reproduced under the terms of the Creative Commons Attribution 4.0 International License (https://creativecommons.org/licenses/by/4.0/). 
was determined that these data did not provide sufficient proof of generalizability of effect across Asian and Western populations.

\section{Using RWE as postmarketing support for an indication approval}

For the past 20 years, adjuvant IFN- $\alpha$-based immunotherapy has been a mainstay of treatment for patients with high-risk resected melanoma [70]. Building on this success, ipilimumab was approved by the FDA in 2015 as adjuvant therapy for fully resected stage III melanoma [71], and nivolumab was approved by both the FDA and the European Commission in 2017/2018 as adjuvant therapy for resected melanoma with involvement of lymph nodes or metastatic disease [72,73]. These regulatory approvals were based on results from Phase III RCTs showing that adjuvant ipilimumab and nivolumab prolonged recurrence-free survival when used within 12 months of resection $[74,75]$. However, there remained at the time a need to assess the benefits of adjuvant I-O therapy on OS among patients with melanoma who progressed to the advanced stages of the disease. As such, a retrospective, longitudinal, EHR-based study was conducted to evaluate real-life survival outcomes among patients with advanced melanoma in relation to whether they had received adjuvant therapy and the type of adjuvant therapy received [76,77].

For the study, data were extracted from a US real-world EHR-derived database for adult patients with advanced cutaneous melanoma (unresectable, stage III-IV) who had $\geq 2$ nonconsecutive clinical encounters between January 2011 and June 2018. This resulted in a final eligible cohort of 1497 patients, of whom 182 had received adjuvant therapy [77]. Fifty-five had received adjuvant therapy with 'new' I-O agents, primarily ipilimumab, nivolumab or pembrolizumab; 65 had received adjuvant therapy with 'older' I-O agents, primarily IFN- $\alpha-2 b$ or peg-IFN- $\alpha-2$ b; and 62 had received adjuvant therapy with non-I-O agents, primarily fluorouracil, vemurafenib, carboplatin/tamoxifen and bendamustine. The remaining 1315 patients had not received adjuvant therapy. Comparisons were made between new I-O agents, older I-O agents and non-I-O agents versus no adjuvant therapy, as well as between both new and older I-O agents versus non-I-O agents; outcome measures were OS and progression-free survival.

This study faced several methodological and data-related challenges. For example, despite starting with a pool of nearly 1500 patients, only 182 had received adjuvant therapy, leading to relatively small sample sizes for the 'adjuvant therapy' subgroups and weakening statistical power of some of the analyses. The small sample sizes may have also contributed to the observed lack of a statistically significant influence of adjuvant therapy on progressionfree survival. An additional contributing factor to this observation may have been the frequent need to use proxies for disease progression under real-world conditions (e.g., the date of 'progression' may in fact be a proxy, such as the date of starting subsequent therapy). Furthermore, the real-world database did not contain complete patient histories and treatment-related details, so there may have been imbalances between the compared subgroups that could have impacted respective survival outcomes.

Despite these challenges, by showing that newer adjuvant I-O therapy was associated with significantly improved long-term survival versus both no adjuvant therapy or non-I-O adjuvant therapy, this research informed the clinical understanding of the risk:benefit assessment of adjuvant I-O therapies among patients progressing to advanced melanoma and assisted in regulatory decision-making.

\section{Conclusion}

In the burgeoning field of I-O, there is a need for rapid regulatory assessment of new and existing treatments in order to expedite patient access in the context of addressing unmet medical need. While these assessments are driven primarily by clinical trial data, there is an increasing role for RWE. However, generation of RWD for regulatory submissions faces challenges, many of which are common across all new drugs and biologics, but some of which are unique to I-O agents.

Building on insights from the reviewed literature and the four case studies described above, we see several key ongoing challenges for the use of RWE in the I-O regulatory process, including small sample sizes, missing/incomplete data, real-world end point definition and availability, and undefined requirements for 'regulatory-grade' RWE. In our cases, the primary methodological and data-related challenges were small sample sizes and missing/incomplete data, with both associated with other limitations typical for RWE studies, such as the potential for unknown population imbalances, confounding factors, misclassification issues and selection bias to influence population-matching methods and outcomes. These challenges represent noteworthy limitations as they increase the level of uncertainty regarding the statistical analyses performed and outcomes observed, and raise questions regarding the generalizability of the findings. The challenge of small sample sizes, while common across many indications, may become more 
prominent for I-O therapies as they are increasingly being studied in rare cancers, with inherently smaller patient populations. Similarly, although missing/incomplete data are also relatively ubiquitous in real-world studies, this limitation, and the associated issues with population matching, may pose a somewhat unique challenge for the I-O field, since I-O agents can be associated with durable survival and evaluation of long-term survival benefits may therefore need to be achieved via real-world research rather than lengthy clinical trials. Importantly, observations from our cases could inform on the key methodological and data-related areas of focus for initiatives aimed at enhancing the role for RWE in the regulatory assessment of I-O therapy. For example, investigating the potential for innovative real-world study designs and end points and novel methodological and/or statistical approaches aimed at limiting the impact of small sample sizes or missing/incomplete data would be of great benefit.

In terms of policy-related challenges, the field would benefit from a greater consensus on what constitutes 'regulatory-grade' RWE. While recent framework documents and guidance have started the process of defining 'regulatory-grade' RWD $[1,34]$, there is a need for greater communication between regulators and industry on reaching benchmarks for acceptable RWE. Agreement between parties on 'approved' methodology, such as acceptable data harmonization techniques, analytical approaches and end points, would be particularly useful for optimizing the utility of RWE in regulatory submissions. In addition, there may also be a need for agreement on how best to ensure integrity and transparency regarding RWD generation. Recommendations by the Real-World Evidence Transparency Initiative Partnership, including the encouragement of real-world study registration and upfront provision of study protocols and statistical analysis plans, are an initial step in the right direction [78]. Endorsement of such processes and procedures by regulatory bodies would, of course, be essential to establishing these processes as best practice.

Thus, while methodological, data-related and policy-related challenges persist, RWE has had, and will continue to have, an important role in regulatory approvals of I-O agents. Our cases highlight some of these challenges and inform on key areas of focus for future research to establish a greater role for RWE. Moreover, the potential lessons learned from the cases described in this article may be applicable to the role of RWE in regulatory assessments of other therapeutic classes or in nononcology indications. Ultimately, increased alignment between regulators and clinical sponsors will be essential for achieving a defined application of RWE in informing development and regulatory processes in the expanding I-O field.

\section{Future perspective}

With enhanced communication and greater alignment between regulators and industry, the hope is that a clear RWE roadmap will emerge in the upcoming years facilitating routine generation of 'regulatory-grade' RWD to support the approval of new I-O therapies. In addition, although certain challenges such as small sample sizes and missing/incomplete data will persist, ongoing and future research into novel study designs, end points, methodologies and statistical approaches should advance our ability to lessen the impact of these limitations on robust RWD generation. Moreover, while the expectation is that data from RCTs will continue to be critical for regulatory appraisal of new I-O agents, continued research on the capacity for duplicating clinical trial results using RWD may inspire an even greater role for RWE in the regulatory process, particularly in therapy areas where RCTs are not feasible or unethical.

\section{Author contributions}

All the authors were involved in the conception of this article, development of the manuscript drafts and approved the final manuscript for publication.

\section{Acknowledgments}

The authors are grateful to the members of the joint Bristol Myers Squibb-Flatiron Healthcare Scientific Advisory Board for their input during the development of this article.

Financial \& competing interests disclosure

All the authors are employees of Bristol Myers Squibb. The authors have no other relevant affiliations or financial involvement with any organization or entity with a financial interest in or financial conflict with the subject matter or materials discussed in the manuscript apart from those disclosed.

Professional writing and editorial assistance was provided by Richard Daniel of Parexel, and was funded by Bristol Myers Squibb. 
Open access

This work is licensed under the Attribution-NonCommercial-NoDerivatives 4.0 Unported License. To view a copy of this license, visit http://creativecommons.org/licenses/by-nc-nd/4.0/

Executive summary

\section{Background}

- Although data from randomized controlled trials are the standard for new drug evaluations, there are situations where conducting a robust clinical trial is not practicable or where the time required to conduct a clinical trial might be seen as an unacceptable delay to patients accessing novel treatments.

- Under these circumstances, there is increasing recognition that real-world evidence (RWE) of effectiveness and safety has an integral role in supplementing and supporting new drug applications.

- Assessing effectiveness and safety in real-world populations may be particularly important when clinical trial results lead to dramatic shifts in standard of care for broad groups of patients, as has been seen recently with the emergence of immuno-oncology (I-O) therapies for numerous cancer types.

RWE \& regulatory assessments of new therapies

- Although the role of RWE in the regulatory process has traditionally been to supplement existing approvals (e.g., for indication expansion), there are several examples where RWE was used to support or supplement new drug approvals, mostly in the rare disease and/or oncology settings.

- However, generation of RWE for regulatory decision-making is subject to various operational, methodological and data-related challenges.

- There is increasing recognition among regulators of the need to address these challenges, and various initiatives are underway in Europe and the USA with the aim of developing novel real-world study designs and end points and improving and standardizing methods and statistical approaches.

Impact of RWE on regulatory policy

- Regulators in Europe and the USA have begun to establish frameworks and industry guidance on the use of RWE in regulatory submissions.

- Nevertheless, there remains a lack of clarity on what constitutes 'regulatory-grade' real-world data (RWD) and an associated need for increased communication between regulatory bodies and those submitting data packages, particularly regarding exactly what is required from the RWD for the related evidence to inform their decisions.

RWE \& the regulatory assessment of I-O agents

- Immunotherapy for cancer is a rapidly expanding therapeutic area; several immune checkpoint inhibitors are already standard of care for multiple tumor types and many more I-O agents are in the development pipeline.

- In this fast-changing therapeutic landscape, there is a need to rapidly assess new I-O agents, which may in some circumstances be better served using RWE, rather than data from randomized controlled trials.

- Nevertheless, generation of RWD on I-O treatments is affected by many of the same challenges as experienced with non-I-O treatment classes.

- Case studies are presented in which RWE on I-O treatments and outcomes were used to support regulatory submissions, each highlighting some of the associated methodological and data-related challenges.

Conclusion

- The reviewed literature and presented case studies highlight several key ongoing challenges for the use of RWE in the $\mathrm{I}-\mathrm{O}$ regulatory process, including small sample sizes, missing/incomplete data, real-world end point definition and availability, and undefined requirements for 'regulatory-grade' RWE.

- Solutions to these challenges revolve around greater alignment between regulators and industry. Through enhanced communication, agreement can be reached on topics such as 'approved' methodologies and end points, integrity and transparency processes during RWE generation, and definitions of 'regulatory-grade' RWE.

\section{References}

Papers of special note have been highlighted as: $\bullet$ of interest; $\bullet \bullet$ of considerable interest

1. US FDA. Framework for FDA's real-world evidence program. (2018). https://www.fda.gov/media/120060/download

-. Initial the US FDA document outlining a framework for a role for real-world evidence (RWE) in regulatory decision-making process.

2. Khosla S, White R, Medina J et al. Real world evidence (RWE) - a disruptive innovation or the quiet evolution of medical evidence generation? F1000Res. 7, 111 (2018).

3. Beaulieu-Jones BK, Finlayson SG, Yuan W et al. Examining the use of real-world evidence in the regulatory process. Clin. Pharmacol. Ther. 107(4), 843-852 (2020).

4. Darrow JJ, Avorn J, Kesselheim AS. FDA approval and regulation of pharmaceuticals, 1983-2018. JAMA 323(2), 164-176 (2020). 
5. Casali PG, Bruzzi P, Bogaerts J, Blay JY. Rare Cancers Europe Consensus Panel. Rare Cancers Europe (RCE) methodological recommendations for clinical studies in rare cancers: a European consensus position paper. Ann. Oncol. 26(2), 300-306 (2015).

6. Sertkaya A, Wong HH, Jessup A, Beleche T. Key cost drivers of pharmaceutical clinical trials in the United States. Clin. Trials 13(2), 117-126 (2016).

7. Martin L, Hutchens M, Hawkins C, Radnov A. How much do clinical trials cost? Nat. Rev. Drug Discov. 16(6), 381-382 (2017).

8. Mahendraratnam N, Eckert J, Mercon K et al. Understanding the need for non-interventional studies using secondary data to generate real-world evidence for regulatory decision making, and demonstrating their credibility. (2019). https://healthpolicy.duke.edu/sites/default/files/atoms/files/non-internvetional_study_credibility_final.pdf

9. Burcu M, Dreyer NA, Franklin JM et al. Real-world evidence to support regulatory decision-making for medicines: considerations for external control arms. Pharmacoepidemiol. Drug Saf. 29(10), 1228-1235 (2020).

10. ElZarrad MK, Corrigan-Curay J. The US Food and Drug Administration's real-world evidence framework: a commitment for engagement and transparency on real-world evidence. Clin. Pharmacol. Ther. 106(1), 33-35 (2019).

11. Christofi T, Baritaki S, Falzone L, Libra M, Zaravinos A. Current perspectives in cancer immunotherapy. Cancers (Basel) 11(10), E1472 (2019).

12. Kerr WG, Chisholm JD. The next generation of immunotherapy for cancer: small molecules could make big waves. J. Immunol. 202(1), 11-19 (2019).

13. Xin Yu J, Hubbard-Lucey VM, Tang J. Immuno-oncology drug development goes global. Nat. Rev. Drug Discov. 18(12), 899-900 (2019).

- Recent minireview describing the global status of immuno-oncology therapy development and expansion.

14. Cave A, Kurz X, Arlett P. Real-world data for regulatory decision making: challenges and possible solutions for Europe. Clin. Pharmacol. Ther. 106(1), 36-39 (2019).

15. European Medicines Agency. Assessment report: yervoy (EMA/603930/2013). (2013). https://www.ema.europa.eu/en/documents/variation-report/yervoy-h-c-2213-ii-0008-epar-assessment-report-variation_en.pdf

16. Center for Drug Evaluation and Research (CDER). Application number 125141 (myozyme): medical reviews. (2006). https://www.accessdata.fda.gov/drugsatfda_docs/nda/2006/125141s0000_Myozyme_MedR.pdf

17. Center for Drug Evaluation and Research (CDER). Application number 125291 (lumizyme): medical reviews. (2010). https://www.accessdata.fda.gov/drugsatfda_docs/nda/2010/125291s000MedR.pdf

18. Center for Drug Evaluation and Research (CDER). Application number 125557 (blinatumomab): medical reviews. (2014). https://www.accessdata.fda.gov/drugsatfda_docs/nda/2014/125557Orig1s000MedRedt.pdf

19. Przepiorka D, Ko CW, Deisseroth A et al. FDA approval: blinatumomab. Clin. Cancer Res. 21(18), 4035-4039 (2015).

20. Gokbuget N, Kelsh M, Chia V et al. Blinatumomab vs historical standard therapy of adult relapsed/refractory acute lymphoblastic leukemia. Blood Cancer J. 6(9), e473 (2016).

21. Sharma V. Pink Sheet - Informa Pharma Intelligence. EMA discusses pivotal role of RWE for six drugs. (2019). https://pink.pharmaintelligence.informa.com/PS125230/EMA-Discusses-Pivotal-Role-Of-RWE-For-Six-Drugs

22. Silverman B. Pink Sheet - Informa Pharma Intelligence. A bakers dozen of US FDA efficacy approvals using real world evidence. (2018). https: //pink.pharmaintelligence.informa.com/PS123648/A-Bakers-Dozen-Of-US-FDA-Efficacy-Approvals-Using-Real-World-Evidence

23. European Medicines Agency. Summary of product characteristics: Zalmoxis. (2016). https://www.ema.europa.eu/en/documents/product-information/zalmoxis-epar-product-information_en.pdf

24. Bipartisan Policy Center. Expanding the use of real-world evidence in regulatory and value-based payment decision-making for drugs and biologics. (2019). https://bipartisanpolicy.org/wp-content/uploads/2019/08/Health_Innovation_Real-World_Evidence_Report_R04.pdf

25. Miksad RA, Samant MK, Sarkar S, Abernethy AP. Small but mighty: the use of real-world evidence to inform precision medicine. Clin. Pharmacol. Ther. 106(1), 87-90 (2019).

26. Miksad RA, Abernethy AP. Harnessing the power of real-world evidence (RWE): a checklist to ensure regulatory-grade data quality. Clin. Pharmacol. Ther. 103(2), 202-205 (2018).

27. Curtis MD, Griffith SD, Tucker M et al. Development and validation of a high-quality composite real-world mortality endpoint. Health Serv. Res. 53(6), 4460-4476 (2018).

28. Hernan MA, Robins JM. Using big data to emulate a target trial when a randomized trial is not available. Am. J. Epidemiol. 183(8), 758-764 (2016).

29. Lodi S, Phillips A, Lundgren J et al. Effect estimates in randomized trials and observational studies: comparing apples with apples. Am. J. Epidemiol. 188(8), 1569-1577 (2019).

30. Gingery D. Real-world evidence: sponsors will need prospective process early in development. (2019). https://pink.pharmaintelligence.in forma.com/PS125232/RealWorld-Evidence-Sponsors-Will-Need-Prospective-Process-Early-In-Development 
31. Sutter S. RWE: comparators, therapeutic area may be key for trial replication. (2019).

https://pink.pharmaintelligence.informa.com/PS140974/RWE-Comparators-Therapeutic-Area-May-Be-Key-For-Trial-Replication

32. Innovative Medicines Initiative (IMI). GetReal initiative. https://www.imi-getreal.eu/

33. European Medicines Agency. Ten recommendations to unlock the potential of big data for public health in the EU. (2020). https://www.ema.europa.eu/en/news/ten-recommendations-unlock-potential-big-data-public-health-eu

34. US FDA. Submitting documents using real-world data and real-world evidence to FDA for drugs and biologics: guidance for industry. (2019). https://www.fda.gov/media/124795/download

\section{- Current FDA guidance for industry on use of RWE in regulatory submissions.}

35. European Medicines Agency. Adaptive pathways workshop. (2016). https://www.ema.europa.eu/en/documents/report/adaptive-pathways-workshop-report-meeting-stakeholders-8-december-2016_en.pdf

36. European Medicines Agency. Guidance for companies considering the adaptive pathways approach. (2016). https://www.ema.europa.eu/ en/documents/regulatory-procedural-guideline/guidance-companies-considering-adaptive-pathways-approach_en.pdf

37. European Medicines Agency. EMA regulatory science to 2025. (2020). https://www.ema.europa.eu/en/documents/regulatory-procedural-guideline/ema-regulatory-science-2025-strategic-reflection_en.pdf

- Overview of EMA strategy for future regulatory processes including desired role for RWE.

38. Sutter S. Pink Sheet - Informa Pharma Intelligence. Real-world evidence: sponsors look to US FDA drug reviews for potential pitfalls. (2019). https://pink.pharmaintelligence.informa.com/PS140937/RealWorld-Evidence-Sponsors-Look-To-US-FDA-Drug-Reviews-F or-Potential-Pitfalls

39. Center for Drug Evaluation and Research (CDER). Application number 207103 (palbociclib): approval package. (2019). https://www.accessdata.fda.gov/drugsatfda_docs/nda/2019/207103Orig1s008.pdf

40. Wedam S, Fashoyin-Aje L, Bloomquist $\mathrm{E}$ et al. FDA approval summary: palbociclib for male patients with metastatic breast cancer. Clin. Cancer Res. 26(6), 1208-1212 (2020).

41. Xin Yu J, Hodge JP, Oliva C, Neftelinov ST, Hubbard-Lucey VM, Tang J. Trends in clinical development for PD-1/PD-L1 inhibitors. Nat. Rev. Drug Discov. 19, 163-164 (2020).

42. Marshall HT, Djamgoz MBA. Immuno-oncology: emerging targets and combination therapies. Front. Oncol. 8, 315 (2018).

43. US FDA. The drug development process. Step 3: clinical research. https://www.fda.gov/patients/drug-development-process/step-3-clinical-research

44. Huang GD, Bull J, Johnston McKee K et al. Clinical trials recruitment planning: a proposed framework from the Clinical Trials Transformation Initiative. Contemp. Clin. Trials 66, 74-79 (2018).

45. Jayasundara K, Hollis A, Krahn M, Mamdani M, Hoch JS, Grootendorst P. Estimating the clinical cost of drug development for orphan versus non-orphan drugs. Orphanet. J. Rare. Dis. 14(1), 12 (2019).

46. Hoering A, Durie B, Wang H, Crowley J. End points and statistical considerations in immuno-oncology trials: impact on multiple myeloma. Future Oncol. 13(13), 1181-1193 (2017).

47. Ferrara R, Pilotto S, Caccese M et al. Do immune checkpoint inhibitors need new studies methodology? J. Thorac. Dis. 10(Suppl. 13), S1564-S1580 (2018)

48. Khozin S, Carson KR, Zhi J et al. Real-world outcomes of patients with metastatic non-small cell lung cancer treated with programmed cell death protein 1 inhibitors in the year following U.S. regulatory approval. Oncologist 24(5), 648-656 (2019).

49. Khozin S, Miksad RA, Adami J et al. Real-world progression, treatment, and survival outcomes during rapid adoption of immunotherapy for advanced non-small cell lung cancer. Cancer 125(22), 4019-4032 (2019).

50. Bair SM, Strelec LE, Feldman TA et al. Outcomes and toxicities of programmed death-1 (PD-1) inhibitors in Hodgkin lymphoma patients in the United States: a real-world, multicenter retrospective analysis. Oncologist 24(7), 955-962 (2019).

51. Ahn BC, Pyo KH, Xin CF et al. Comprehensive analysis of the characteristics and treatment outcomes of patients with non-small cell lung cancer treated with anti-PD-1 therapy in real-world practice. J. Cancer Res. Clin. Oncol. 145(6), 1613-1623 (2019).

52. Arheden A, Skalenius J, Bjursten S et al. Real-world data on PD-1 inhibitor therapy in metastatic melanoma. Acta Oncol. 58(7), 962-966 (2019).

53. Guisier F, Dubos-Arvis C, Vinas F et al. Efficacy and safety of anti-PD-1 immunotherapy in patients with advanced non small cell lung cancer with BRAF, HER2 or MET mutation or RET-translocation. GFPC 01-2018. J. Thorac. Oncol. 15(4), 628-636 (2020).

54. Kirchberger MC, Moreira A, Erdmann M, Schuler G, Heinzerling L. Real world experience in low-dose ipilimumab in combination with PD-1 blockade in advanced melanoma patients. Oncotarget 9(48), 28903-28909 (2018).

55. Cortellini A, Buti S, Santini D et al. Clinical outcomes of patients with advanced cancer and pre-existing autoimmune diseases treated with anti-programmed death-1 immunotherapy: a real-world transverse study. Oncologist 24(6), e327-e337 (2019).

56. European Medicines Agency. Assessment report: bavencio (EMA/496529/2017). (2017). https://www.ema.europa.eu/en/documents/assessment-report/bavencio-epar-public-assessment-report_en.pdf 
57. Merck KGaA and Pfizer Inc. European Commission approves bavencio (avelumab) for metastatic Merkel cell carcinoma. (2017). https://www.pf izer.com/news/press-release/press-release-detail/european_commission_approves_bavencio_avelumab_for_metastatic_mer kel_cell_carcinoma

58. Kaufman HL, Russell J, Hamid O et al. Avelumab in patients with chemotherapy-refractory metastatic Merkel cell carcinoma: a multicentre, single-group, open-label, Phase II trial. Lancet Oncol. 17(10), 1374-1385 (2016).

59. Iyer JG, Blom A, Doumani R et al. Response rates and durability of chemotherapy among 62 patients with metastatic Merkel cell carcinoma. Cancer Med. 5(9), 2294-2301 (2016).

60. Edwards SJ, Wakefield V, Cain P, Kew KM, Salih F. BMJ Technology Assessment Group. Avelumab for treating metastatic Merkel cell carcinoma: a single technology appraisal. (2017). https://www.google.ie/url?sa=t\&rct=j\&q=\&esrc=s\&source=web\&cd=5\&ved=2ahUK EwjV0Jjw9OnnAhULXxoKHarHBQMQFjAEegQIAxAB\&url=https\%3A\%2F\%2Fnjl-admin.nihr.ac.uk\%2Fdocument\%2Fdownloa d\%2F2022737\&usg=AOvVaw1nOu98q5GhV9f 1IqWCyyy

61. Bristol Myers Squibb. US Food and Drug Administration approves Opdivo ${ }^{\circledR}$ (nivolumab) as the first new medication in nearly 20 years for certain patients with previously treated small cell lung cancer. (2018).

https://news.bms.com/press-release/corporatefinancial-news/us-food-and-drug-administration-approves-opdivo-nivolumab-firs

62. Cope S, Popoff E, Keeping $S$ et al. Comparative efficacy of nivolumab \pm ipilimumab versus standard of care (SOC) for third-line (3L) patients with recurrent small cell lung cancer (SCLC) using population-adjusted indirect comparison. Value Health 21(Suppl. 3), S18 (2018).

63. Cope S, Popoff E, Keeping ST et al. Comparative efficacy of nivolumab \pm ipilimumab vs. standard of care for third-line patients with recurrent small cell lung cancer using population-adjusted indirect comparison. Presented at: ISPOR Europe 2018. Barcelona, Spain, 10-14 November 2018 (Poster).

64. Reck M, Vicente D, Ciuleanu T et al. Efficacy and safety of nivolumab (nivo) monotherapy versus chemotherapy (chemo) in recurrent small cell lung cancer (SCLC): results from CheckMate 331. Ann. Oncol. 29, x43 (2018).

65. National Comprehensive Cancer Network. NCCN clinical practice guidelines in oncology: small cell lung cancer - version 1.2021. (2020). https://www.nccn.org/professionals/physician_gls/pdf/sclc.pdf

66. Kang YK, Boku N, Satoh T et al. Nivolumab in patients with advanced gastric or gastro-oesophageal junction cancer refractory to, or intolerant of, at least two previous chemotherapy regimens (ONO-4538-12, ATTRACTION-2): a randomised, double-blind, placebo-controlled, Phase III trial. Lancet 390(10111), 2461-2471 (2017).

67. Bristol Myers Squibb. Japan Ministry of Health, Labor and Welfare approves Opdivo (nivolumab) for the treatment of patients with unresectable advanced or recurrent gastric cancer which has progressed after chemotherapy. (2017). https://news.bms.com/press-release/corporatefinancial-news/japan-ministry-health-labor-and-welfare-approves-opdivo-nivolu

68. Ott P, Emiliano C, Sharma P et al. Nivolumab monotherapy in patients with advanced gastric or gastroesophageal junction (GEJ) cancer and 2 or more prior treatment regimens: sub-analysis of the CheckMate 032 study. Ann. Oncol. 28(Suppl. 3), iii139 (2017).

69. Chau I, Le DT, Ott PA et al. Developing real-world comparators for clinical trials in chemotherapy-refractory patients with gastric cancer or gastroesophageal junction cancer. Gastric Cancer 23(1), 133-141 (2020).

70. Achkar T, Tarhini AA. The use of immunotherapy in the treatment of melanoma. J. Hematol. Oncol. 10(1), 88 (2017).

71. Bristol Myers Squibb. Bristol-Myers Squibb receives approval from the U.S. Food and Drug Administration for Yervoy (ipilimumab) as adjuvant treatment for fully resected stage III melanoma. (2015).

https://news.bms.com/press-release/financial-news/bristol-myers-squibb-receives-approval-us-food-and-drug-administration-

72. Bristol Myers Squibb. Bristol-Myers Squibb receives FDA approval for Opdivo (nivolumab) as adjuvant therapy in patients with completely resected melanoma with lymph node involvement or metastatic disease. (2017).

https://news.bms.com/press-release/corporatefinancial-news/bristol-myers-squibb-receives-fda-approval-opdivo-nivolumab-ad

73. Bristol Myers Squibb. European Commission approves Bristol-Myers Squibb's Opdivo (nivolumab) for the adjuvant treatment of adult patients with melanoma with involvement of lymph nodes or metastatic disease who have undergone complete resection. (2018). https://news.bms.com/press-release/corporatefinancial-news/european-commission-approves-bristol-myers-squibbs-opdivo-ni-0

74. Eggermont AM, Chiarion-Sileni V, Grob JJ et al. Adjuvant ipilimumab versus placebo after complete resection of high-risk stage III melanoma (EORTC 18071): a randomised, double-blind, Phase III trial. Lancet Oncol. 16(5), 522-530 (2015).

75. Weber J, Mandala M, Del Vecchio M et al. Adjuvant nivolumab versus ipilimumab in resected stage III or IV melanoma. N. Engl. J. Med. 377(19), 1824-1835 (2017).

76. Gu S, Le T, Moshyk A, Gooden K, Kotapati S. Adjuvant therapy is associated with improved overall survival in advanced melanoma patients in the United States. Pigment Cell Melanoma Res. 32(1), 140 (2019).

77. Gu S, Le T, Moshyk A, Gooden K, Kotapati S. Adjuvant therapy is associated with improved survival in advanced melanoma patients in the United States. Presented at: 15th International Congress of the Society for Melanoma Research. Manchester, UK, 24-27 October 2018 (Poster).

78. The Real-World Evidence Transparency Initiative Partnership Steering Committee. Improving transparency in non-interventional research for hypothesis testing - why, what, and how: considerations from the real-world evidence transparency initiative. (2019). 
https://www.ispor.org/docs/default-source/strategic-initiatives/improving-transparency-in-non-interventional-research-for-hypothesis -testing_final.pdf?sfvrsn=77fb4e97_6

- Recent draft white paper on how to improve integrity and transparency in relation to real-world studies and associated RWD/RWE. 